\title{
The first frontier: High altitude ballooning as a platform for student research experiences in science and engineering
}

\author{
Shane L. Larson ${ }^{\text {a) }}$ \\ Department of Physics, Utah State University, Logan, Utah 84322 \\ John C. Armstrong ${ }^{\text {b) }}$ \\ Department of Physics, Weber State University, Ogden, Utah 84408 \\ William A. Hiscock ${ }^{\mathrm{c})}$ \\ Department of Physics, Montana State University, Bozeman, Montana 59717
}

(Received 26 November 2008; accepted 19 February 2009)

\begin{abstract}
High altitude balloon platforms are ideal for providing hands-on research experiences for students in physics, atmospheric science, engineering, and other aerospace-related disciplines. We describe a basic high altitude balloon platform that can be constructed and operated by undergraduate students. The existing HARBOR and BOREALIS programs are used to illustrate some possible science and engineering research projects that students can pursue as part of a high-altitude flight program. (C) 2009 American Association of Physics Teachers.

[DOI: 10.1119/1.3097775]
\end{abstract}

\section{INTRODUCTION}

The aging of the technological workforce ${ }^{1-3}$ and the declining student interest in STEM fields ${ }^{4}$ have produced many efforts to bring science and technology into university and college classrooms and provide students with exciting hands-on experience to stimulate their interest. The lure of traveling and working "in space" is arguably one of the most prominent drivers for students interested in aerospace-related disciplines, and this paper describes a way for building on those interests.

High altitude ballooning uses commercial, off-the-shelf hardware to launch student experiments with typical maximum altitudes ranging from 30 to $35 \mathrm{~km}$. As a research experience for students, high altitude ballooning provides direct experience in the design, construction, and flight of hardware destined for the space environment. At typical altitudes reached by these systems, experiments are subject to conditions unlike any experienced on the ground (extreme cold, very low pressure, and high cosmic radiation). Although not the ultra-high vacuum of space, the conditions at these altitudes are similar to those encountered on the surface of the planet Mars. At an altitude of $35 \mathrm{~km}$, the regime targeted by high altitude balloons, the standard atmospheric model $^{5}$ gives pressures of $P=560 \mathrm{~Pa}(0.006 \mathrm{~atm})$, temperatures of $T=-36{ }^{\circ} \mathrm{C}$, and densities of $\rho=0.008 \mathrm{~kg} / \mathrm{m}^{3}$. Student-designed flight systems that reach these altitudes must be capable of operating in these extreme conditions, and must survive the journey back down to the surface of Earth for recovery, analysis, and eventual reflight. Engineering robust flight systems within tight constraints on mass and power is only part of a high altitude ballooning program; scientific experiments also play a prominent role, with opportunities ranging from probes of the structure and composition of the atmosphere, to remote sensing with Earth observing experiments, and measurements of cosmic radiation.

High altitude ballooning is not a new endeavor, and has long been an amateur hobby with significant participation from the amateur radio community. Many resources exist online documenting the development of a high altitude balloon system (see, for example, Refs. 9-11). Because the systems are designed from off-the-shelf hardware, many options exist for the different subsystems, whether those subsystems are purchased assembled, or are designed and constructed from readily available components by a student team. Each balloon system is unique and reflects the engineering and budgetary constraints of the team that builds it. There is no definitive way to construct a system, but the basics are shared by most high altitude balloon systems; those basics are described here.

In this paper the basic design and implementation of a student-centered high altitude ballooning program is described, using the HARBOR (High Altitude Reconnaissance Balloon for Outreach and Research) program at Weber State University and the BOREALIS (Balloon Outreach, Research, Exploration And Landscape Imaging System) program at Montana State University as examples.

The flight of high altitude balloons is regulated by the Federal Aviation Administration. ${ }^{6}$ The FAA regulations are subject to revision, and a critical step of the mission planning process is consulting these regulations regularly. Balloons carrying small, low density payloads may be exempt from regulation. $^{7}$

The basic hardware is described in Sec. II, and flight operations are described in Sec. III. The principles of flight analysis are discussed in Sec. IV, and science and engineering are covered in Sec. V. Reflections on the continuing development of high altitude ballooning in university settings and the value it brings to the undergraduate experience are discussed in Sec. VI.

\section{FLIGHT HARDWARE}

A variety of constraints on high altitude balloon design and operations exist in the FAA regulations, but the most important one is the mass limits: $12 \mathrm{lb}(5.44 \mathrm{~kg})$ total, with no single element weighing more than $6 \mathrm{lb}(2.72 \mathrm{~kg})$. These constraints are an important facet of the research experience, providing a clear and well defined set of limits to which students' designs must conform, allowing them to exercise their skills and ingenuity while practicing the real-world exercise of design trade-offs in the development of spaceflight hardware. 


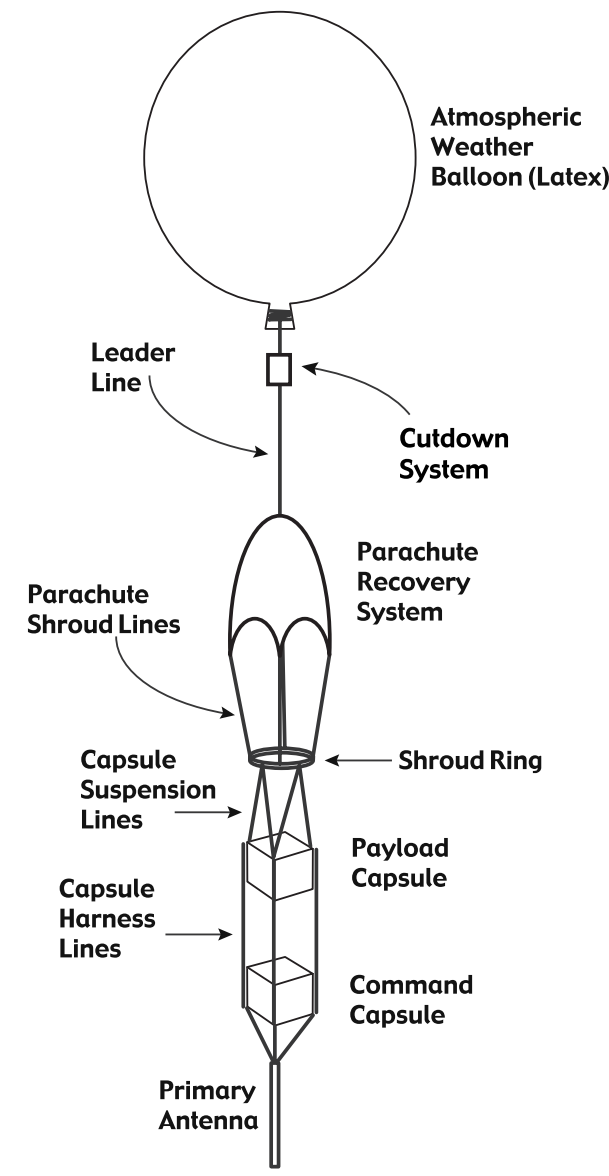

Fig. 1. The elements of a typical high altitude balloon stack.

The core elements of a standard high altitude balloon system are shown in Fig. 1. A basic balloon system is built around a commercially available weather balloon filled with helium to carry a payload aloft. ${ }^{8}$ Weather balloons are typically latex rubber and are sold according to mass. Heavier (thicker) latex balloons can expand more and hence burst at higher altitudes. For the balloon systems described in this paper, 1200-2000 g balloons are typical. The balloon is attached to the rest of the balloon stack via a single leader line. The FAR requires two operational cut-down systems, a timed cut-down and a remote control "abort" cut-down, which can be accomplished using a nickel-chromium wire wrapped around the leader line to the balloon. When the cut-down circuit is engaged, the wire burns through the lead line, severing the contact between the payload and the balloon.

The leader line connects to the top of a large $(\approx 2 \mathrm{~m})$ diameter nylon parachute that is the primary recovery device

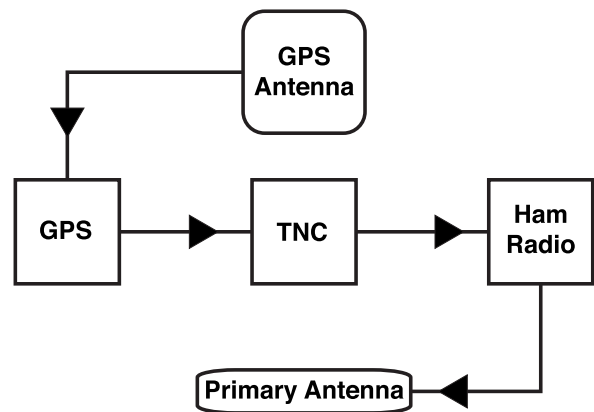

Fig. 2. A simple block diagram of a basic high altitude balloon telemetry system. The GPS feeds the location and altitude into the terminal node controller (TNC) that encodes the information into APRS packets that it feeds to the radio for transmission to the ground.

for the system after the balloon bursts. A variety of companies offer commercial parachutes for use by the amateur high power rocket community, and it is easy to sew your own. Some teams use a lightweight wooden ring to help keep the parachute shroud-lines from fouling during the flight, and to connect the parachute to the uppermost payload capsule.

Payload capsules are sized to suit the experiments and equipment they carry, and are typically $\approx 30 \mathrm{~cm}$ cubes. Commercial styrofoam packing boxes may be used, or custom boxes constructed from high density polystyrene foam readily available at home improvement stores. The capsules are shrouded in ripstop nylon covers to make them highly visible and provide connection points for the harness lines that hold them in the stack. The foam boxes can be easily cut to allow experimental access to the outside environment (such as probes, cameras, and antennae). One payload capsule carries the primary radio tracking system. The basic block diagram of this system is shown in Fig. 2, consisting of a GPS receiver and an encoding system to package the GPS data in APRS (Automatic Packet Reporting System) format and feed it to an amateur transmitter, which broadcasts the data to the ground via the primary antenna. A handheld or mobile radio on the ground then receives several data packets every minute, each one giving the balloon's present latitude, longitude, and altitude. Teams design their own system based on their approach to hardware engineering. Table I illustrates the two different solutions to the block diagram in Fig. 2 taken by the BOREALIS and HARBOR teams.

Redundancy is an important part of space hardware design. Although a high-altitude balloon science package can fail with small implications for a flight team (generally the cost of a reflight), the failure of the primary tracking system is intolerable because it will almost certainly lead to the loss of the balloon system, and an untracked balloon aloft is a

Table I. The hardware for the primary comm systems for BOREALIS and HARBOR. The two programs have developed their own communications solutions from off the shelf hardware.

\begin{tabular}{lll}
\hline \hline Block element & BOREALIS & HARBOR \\
\hline GPS Antenna & Garmin 25MCX & Garmin 25MCX \\
GPS Chip & Garmin 15H-W & Garmin GPS 15LW \\
TNC (Terminal Node Controller) & Built into Kenwood TH-D7 & Built into Microtrak 8000 \\
Radio & Kenwood TH-D7AG & Byonics Microtrak 8000FA \\
Antenna & $1 / 4$-wave whip with ground plane & $1 / 4$-wave whip \\
\hline \hline
\end{tabular}


Table II. An example mass budget from HARBOR. CATS is the Command And Telemetry System, BATS is the Balloon Auxiliary Tracking System, ARIES is the digital imaging system, and PASCAL is an integrated pressure/ temperature sensor. FAA regulations on the mass of each payload box is 6 pounds; the listed HARBOR limit is the 6 pound FAA limit, less the mass of the flight and recovery systems (split between the two payloads). Unless otherwise noted, individual sensors and experiment masses include battery masses.

\begin{tabular}{|c|c|c|}
\hline & Mass (g) & Mass (pounds) \\
\hline \multicolumn{3}{|c|}{ Flight system } \\
\hline Parachute & 461.0 & 1.016 \\
\hline Cutdown system & 207.7 & 0.458 \\
\hline Flight system total & 668.7 & 1.474 \\
\hline \multicolumn{3}{|c|}{ Command/control payload } \\
\hline CATS (with batteries) & 706.0 & 1.556 \\
\hline BATS (with batteries) & 688.6 & 1.518 \\
\hline ARIES & 228.5 & 0.504 \\
\hline Box, bag, lines & 484.7 & 1.069 \\
\hline Aux temp sensor & 63.6 & 0.140 \\
\hline Cmd-Ctrl total & 2171.4 & 4.787 \\
\hline Cmd-Ctrl limit & 2387.2 & 5.263 \\
\hline Cmd-Ctrl margin & -215.8 & -0.476 \\
\hline \multicolumn{3}{|c|}{ Science payload } \\
\hline PASCAL & 226.0 & 0.498 \\
\hline Box, bag, lines & 484.7 & 1.069 \\
\hline Science total & 710.7 & 1.567 \\
\hline Science limit & 2387.2 & 5.263 \\
\hline Science margin & -1676.5 & -3.696 \\
\hline System total & 3550.8 & 7.828 \\
\hline System limit & 5.443 .1 & 12.000 \\
\hline System margin & -1892.3 & -4.172 \\
\hline
\end{tabular}

navigation hazard during the time it traverses the altitudes of normal air traffic. For this reason, both BOREALIS and HARBOR carry fully independent secondary tracking systems that broadcast the GPS location at a lower cadence and at slightly lower power. The lower power requirement means the system will typically operate for a day or more, so that if a balloon is grounded, it will transmit its location long enough for a recovery team to find it.

Power supplies (typically batteries) will be subjected to extreme temperatures, and some batteries (notably common alkalines) perform very poorly at cold temperatures. Once a student flight team has designed a system that requires power, it is important to test any battery system on the ground under cold conditions prior to flight.

Every component of the system contributes to the overall mass of the system. Mass is probably the most important design constraint placed on student flight teams, because some hardware is essential (like tracking and communications), and science hardware must fit within the left over mass margins. A typical mass budget for the HARBOR system is shown in Table II. It is not necessary for teams to fly at full mass; flight teams will often find that their experimental payloads will have a mass well below the limits allowed by the FAR. Flying low mass systems requires less helium. In some instances, student flight teams will deliberately fly with less payload, because less helium on average means

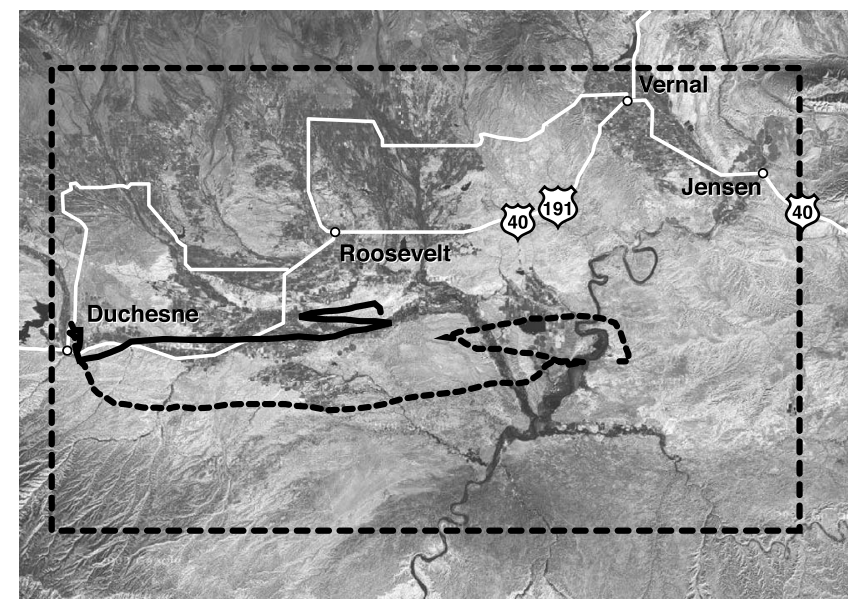

Fig. 3. A typical HARBOR flight prediction (solid black line) and actual flight data (dashed black line) in the Uintah Basin of eastern Utah, launching from Duchesne. The outer dashed box is the allowed flight range accepted by the student flight team; beyond its boundaries is considered too far for recovery operations $(\approx 100 \mathrm{~km}$ down range in this case) or the terrain is considered too inaccessible to guarantee successful recovery of the flight hardware. Maps and tracks produced by Google Earth.

slow ascents with higher burst altitudes. Teams that pursue this method to see how high they can fly should be ready for a long downrange recovery.

\section{FLIGHT OPERATIONS}

\section{A. Flight prediction}

The first step in preparing for a balloon flight is predicting the expected trajectory of the balloon. Flight predictions are made based on atmospheric soundings from the Global Forecast System, provided daily by the National Oceanic and Atmospheric Administration. ${ }^{12}$ Many options exist, though the most commonly used are the Windows-based Balloon Track software, ${ }^{13}$ and the web-based Near Space Flight Track Prediction Utility. ${ }^{14}$ These programs take as input the expected rise rate of the balloon (typically $150-300 \mathrm{~m} / \mathrm{min}$ ), the expected descent rate, the estimated burst altitude, and the latitude and longitude of the launch. The predicted flight track allows the flight team to assess if the weather on a given day is favorable for a launch, and whether the balloon will track into terrain or other geographical areas that are unfavorable for a successful recovery. It is important to be aware of the limitations of flight prediction and the differences between different software tools. Balloon Track uses direct atmospheric samples collected from NOAA sites around the country ("sounding data") to make flight predictions for the exact wind profile in the data. In contrast, the Near Space Ventures tool ${ }^{14}$ uses modeled profiles derived from sounding data, which might provide more reliable flight tracks from a balloon team's actual launch site. Flight predictions should be treated and used in flight planning with care.

The BOREALIS and HARBOR teams develop flight predictions using a flight range box that covers the areas the team is willing to recover the balloon from (in terms of terrain and distance the teams can drive during the chase). The HARBOR flight range box is shown in Fig. 3, along with typical predicted and resulting flight tracks. In the Rocky Mountain area, most balloon flights track from the west to 
the east with the prevailing winds, so the launch site is located on the western edge of the box. If a flight track prediction lands in the box, conditions are deemed suitable for a launch; otherwise, the conditions are unfavorable for a launch. Other ballooning programs, such as that at Medgar Evers College ${ }^{15}$ and at the University of Puerto Rico have geographical challenges to payload recovery that greatly exceed those of the Rocky Mountains.

\section{B. Preflight preparations}

Hardware preparations are unique to each high altitude balloon system. Each piece of hardware needs to be inspected, tested, and prepared before each flight. The procedures for preparation are carried out by following detailed checklists which the student hardware team prepares during development and testing. These checklists are printed and archived for use during each flight, and often go through several revisions during the testing phase. They include every step required to prepare a balloon hardware subsystem, including attachment to the balloon capsules, attachment of wires and cables, insertion of batteries, power-up procedures, and final operations verification.

It is essential that the student flight team adhere to the checklist procedures, and adopt it as an integral part of the launch culture. The checklists are implemented to minimize risk to the team and hardware, and to maximize the chances of success and the return of scientific data. Preflight checklists typically involve one-week preflight, one-day preflight, and flight-line procedures. The one-week preflight checklists, in addition to hardware preparation, also typically cover required communications with the FAA, as well as preparations relating to the college or university (such as reserving vehicles and contacting the press office).

\section{Launch operations}

All that is required for a launch site is a relatively flat site free of surrounding high trees, power or communications lines, and other obstructions to flight. The ramp area of a small airport may be ideal. BOREALIS and HARBOR have often benefited from an offer to stage operations or inflate their balloons inside an empty hangar on a windy morning. Small airports also offer some advantages in communicating with the FAA, because their locations are well established and are known centers for flight activity.

Launches are usually done near dawn to reduce the effect of surface winds which build as the sun rises. At the launch site the balloon is unfurled on a clean linen sheet on the ground to prevent small sticks and stones from harming the balloon. Team members handling the balloon wear cotton gloves to prevent fingernails and skin oils from weakening or damaging the latex. Custom fill nozzles may be constructed easily from PVC pipe fittings to fit snugly inside the neck of the balloon while filling it with helium; typically two $\mathrm{K}$ size tanks of helium are needed for a full fill. The amount of helium needed depends on the overall payload being carried aloft. To get the standard rise rates of $150-300 \mathrm{~m} / \mathrm{min}, \mathrm{BO}$ REALIS and HARBOR fill until the balloon provides $120 \%$ to $150 \%$ of the payload weight in lift. The lift is measured in the field by attaching the partially filled balloon to a gym-bag ballasted with the desired mass in water bottles; helium is continually pumped into the balloon until it can lift the bal-

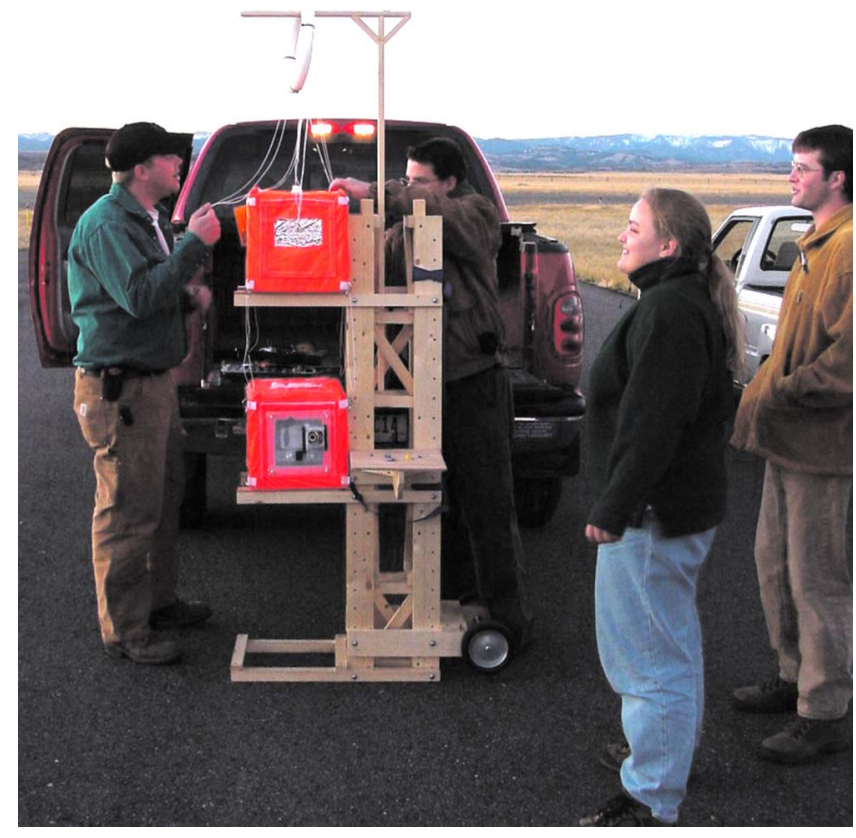

Fig. 4. The BOREALIS launch tower, with payload boxes being prepped for a flight by the student flight team.

last bag, at which time it is tied off by looping one end of the leader line over the neck of the balloon, folding it over, and applying a liberal amount of duct tape.

The fill procedure generally takes about 30 min. During the fill, part of the student flight team prepares the capsules and hardware, following the checklists. The BOREALIS and HARBOR programs utilize wooden launch towers, which have shelves to hold the payload capsules off the ground. The launch tower allows the team to hook up harness lines without tangling and to easily work on installing and turning on experiments and communications hardware inside the modules. The BOREALIS launch tower is shown on the flightline in Fig. 4.

\section{Flight, chase, and recovery}

A schematic of the typical flight profile is shown in Fig. 5. The balloon is not released until it has been verified that both the primary and backup tracking systems are regularly broadcasting the GPS location of the balloon, which can be received by amateur radio operators on the ground and by amateur radio "digipeaters," which pick up the signal and rebroadcast it. The chase team monitors the location and any other broadcast telemetry from the ground. Reports on the latitude, longitude, and altitude are called in to the FAA during ascent and descent through the flight levels; air traffic control (ATC) is usually very explicit (and helpful) in specifying what events (for example, altitude crossings) they would like reported.

During the ascent the track of the balloon can be plotted; the chase team endeavors to stay as close to underneath the balloon as possible or, if roads are scarce, to be in the vicinity of the likely landing locale. On HARBOR and BOREALIS flights, several members of the student flight team immediately begin using the returned GPS data after launch to determine the balloon's rise rate, and flight simulations are rerun with the on-flight data to provide updated predictions for the balloon's flight track. If the team is large enough, it is 


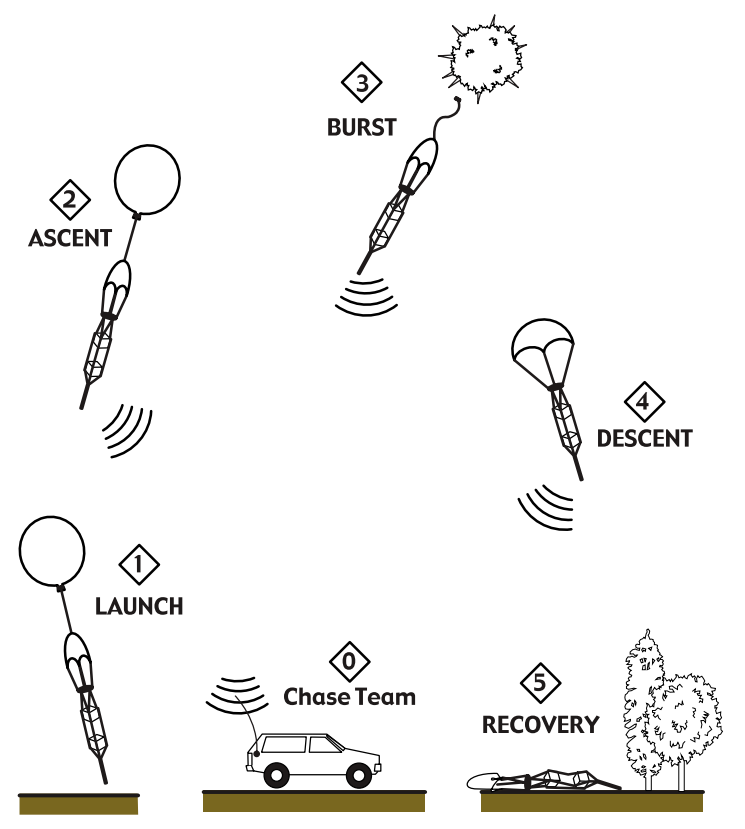

Fig. 5. A typical high altitude balloon flight profile.

possible to dispatch a separate recovery team to the area of the anticipated landing site before launch takes place.

As the balloon ascends the atmospheric pressure decreases, and the balloon expands. It eventually expands past the breaking point of the latex, and the balloon bursts and begins to descend; on a clear day, the balloon may be visible from the ground until the time when it bursts. The descent begins quite rapidly due to the near vacuum, but as the atmosphere becomes thicker at lower altitudes, the parachute slows the descent to a nominal value of $4-5 \mathrm{~m} / \mathrm{s}$.

Once the balloon comes to rest on the ground, the chase team must recover it. Ideally, the team will receive GPS data directly from the balloon on the ground. Often a team must start from the last known GPS coordinates, typically returned from an altitude of several hundred meters. It is important to be close to the landing site when the balloon makes its final descent, because proximity to the downed balloon will often allow a weak or obscured signal to be received by the chase team, ultimately getting them to the final landing site. Use of directional antennae and radio direction finding skills can also be useful if only partial APRS packets are received due to terrain complications.

Landowners should be contacted for permission to enter or cross their property during the recovery operations. The flight team should be prepared to hike to the downed balloon if roads cannot get them in close proximity, as was the case for the recovery shown in Fig. 6.

By using these methods, the BOREALIS program has successfully flown over 60 missions with a $100 \%$ payload recovery record since the first flight in 2001. HARBOR has drawn from the BOREALIS heritage to develop a comparable system that has its own unique features and was successfully flown during the 2007-2008 flight season.

\section{FLIGHT ANALYSIS}

The analysis of balloon flights, both pre- and postflight, provides many opportunities for students to exercise skills that are commonly taught in undergraduate science curricula

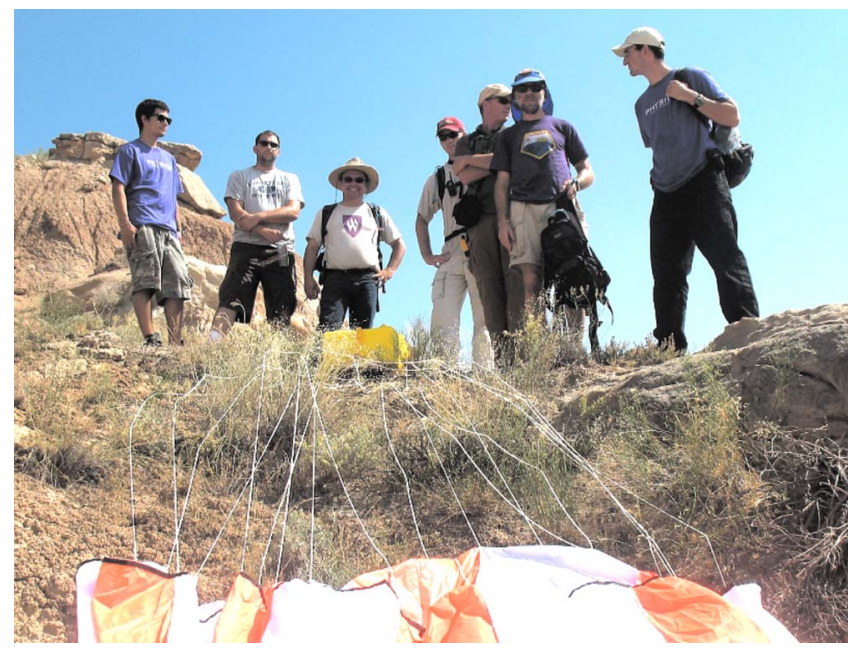

Fig. 6. The HARBOR flight team at a balloon recovery on August 2008. The terrain in recovery zones can be rugged, like that pictured here.

(see, for example, Ref. 16, an analysis of ascent for cluster ballooning). A basic approach ${ }^{17,18}$ for uniform atmospheres is to analyze the dynamics by balancing the forces on the balloon: the drag, the buoyancy, and the weight.

High altitude balloons like HARBOR and BOREALIS traverse a large portion of the atmosphere, which is not uniform over the duration of the flight. A typical altitude profile for a sounding balloon flight is shown in Fig. 7. Working out the altitude trajectory from first principles is very difficult, ${ }^{19,20}$ because it requires a detailed model of the atmosphere and a model of the physical character of the balloon, ${ }^{21}$ which changes constantly during the flight as a consequence of the amount of helium used in the fill and the material properties of the balloon. The ascent dynamics are further complicated by the fact that the balloon transitions from turbulent fluid flow through the atmosphere at low altitudes, to smooth laminar flow at higher altitudes. Descent dynamics are dominated by the character of the parachute used for the recovery system. BOREALIS uses a four-panel

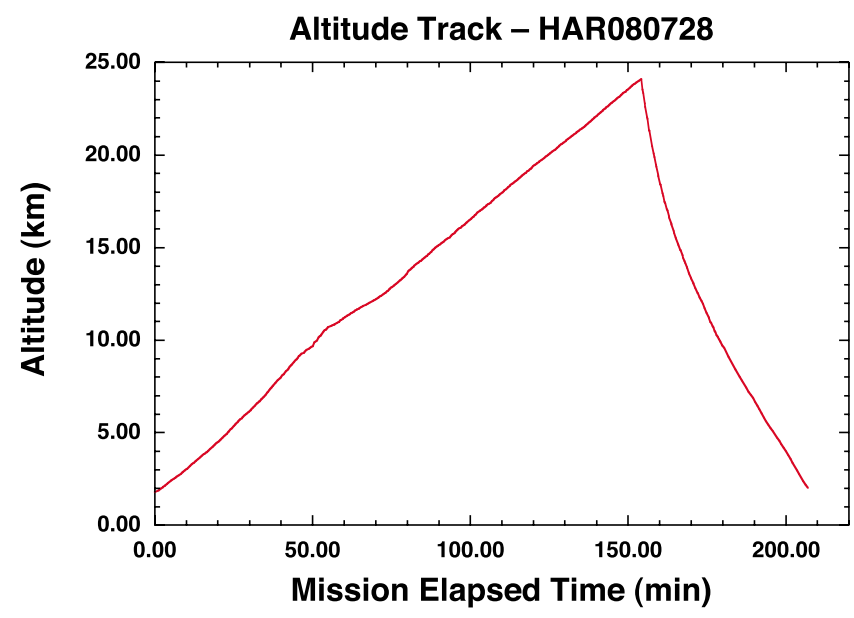

Fig. 7. A typical altitude profile generated by returned GPS data from HARBOR in August 2008. The balloon rapidly reaches terminal velocity during ascent, and maintains a nearly linear climb until burst. The descent is initially rapid, as the atmosphere at burst altitude is too thin for effective braking. 
parachute with rhomboid gores that spills air out of the wedges where the individual gores meet on two sides. HARBOR uses a hemispherical parachute with a spill-hole at the apex that spills air directly out of the top of the parachute. These two parachutes have different inflation, drag, and different oscillation properties as the stack descends, which affects the overall descent profile.

A classic activity carried out by the student flight team is curve fitting the balloon track. This approach to flight analysis circumvents the difficulty of deriving a theoretical shape for the flight trajectory. Fits to the ascent and descent profiles can be fitted and used for future flight predictions. For the profile shown in Fig. 7 a fit of the rise rate to a linear function yields $2.44 \mathrm{~m} / \mathrm{s}$. The descent profile shown in Fig. 7 is well fit by an exponential function $\exp (-0.04 t)$, where $t$ is the elapsed time since burst in minutes.

One of the basic problems of flight analysis is that most experiments record data versus time, but what is usually of interest is data versus altitude. The altitude information is encoded in the GPS track of the balloon system and is not recorded at exactly the same points as the data in individual experimental subsystems. A microprocessor-controlled telemetry system that will take the GPS signals and immediately merge them with the experimental data streams can be implemented, but the simplest systems to implementcharacteristic of a beginning program-will utilize simple data loggers to record experimental results rather than telemetry. An early analysis project for students is to develop codes for correlating experimental data records with the flight record, and interpolating altitude points that correspond with the raw scientific data. Each of the graphs shown in this paper was reduced using a cubic-spline interpolation ${ }^{22}$ of the altitude track from the balloon's GPS data.

\section{BALLOON SCIENCE AND ENGINEERING}

The types of experiments that can be carried aloft are limited only by the constraints of the balloon hardware and the imagination of the student team. The most limiting factor is generally mass, but creative applications of technology will often reduce the size and mass of a given experiment. For many physical quantities, commercial data loggers and sensors suitable for high altitude balloon flights are readily available so that telemetry is unnecessary to capture the science data (assuming the payload is recovered). This section discusses several examples of student experiments that have been flown on BOREALIS and HARBOR.

\section{A. Temperature profiles}

The vertical structure of the atmosphere is differentiated by temperature. High altitude balloons traverse the troposphere and fly into the stratosphere. The temperature decreases in the troposphere as altitude increases, then levels off at the transition into the stratosphere; this transition is called the tropopause. Ascending through the stratosphere, the temperature begins to rise again because the atmosphere is heated from above by the absorption of ultraviolet light by ozone in the upper reaches of the stratosphere. A typical temperature profile from a high altitude balloon flight is shown in Fig. 8, showing the passage of the balloon through the tropopause on ascent and descent.

Many balloon teams fly more than one temperature sensor, monitoring both the internal and external temperature of

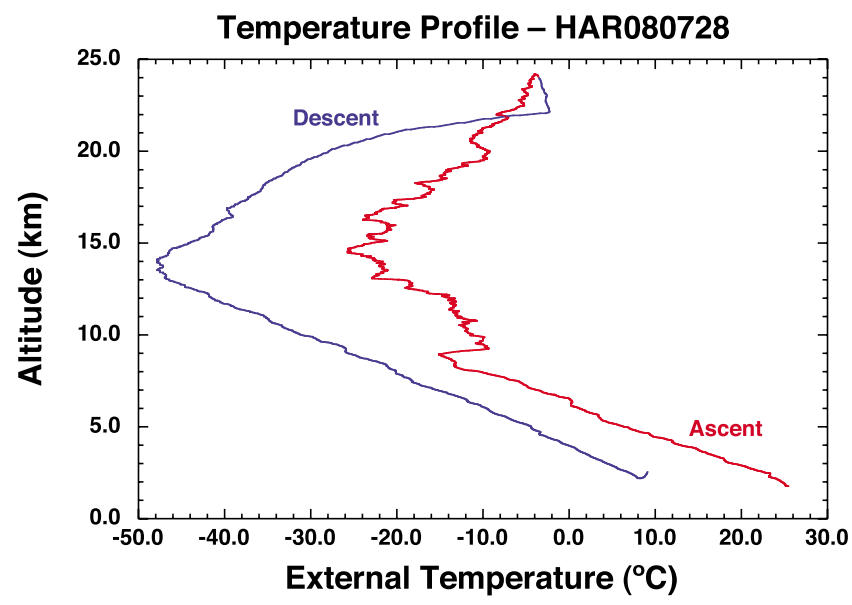

Fig. 8. An atmospheric temperature profile collected by HARBOR in August 2008. Note that the tropopause is identifiable in both the ascent and descent profiles at around $14 \mathrm{~km}$. The difference between ascent and descent values is likely due to condensation on the temperature probe. Improving sensor performance and mitigating biasing effects, such as condensation, are subjects of future student work.

their payload modules. This information provides an opportunity to understand the thermal character of the payload boxes, as well as the heat generated by other experiments carried in the modules, providing useful data for designing future experimental payloads.

\section{B. Pressure profiles}

One of the defining characteristics of "outer space" is the lack of an atmosphere, which can easily be measured in terms of the atmospheric pressure. The Standard Atmosphere $^{5}$ at $35 \mathrm{~km}$ altitude is $P=560 \mathrm{~Pa}$, comparable to the surface pressure on Mars. Pressure profiles for the ascent and descent of a typical flight are shown in Fig. 9, plotted against the Standard Atmosphere. The Standard Atmosphere is a model for the structure of the atmosphere and covers regions of the atmosphere well above those accessible to high altitude balloons. In the lower reaches of the atmosphere the pressure can be calculated from a simple exponential model,

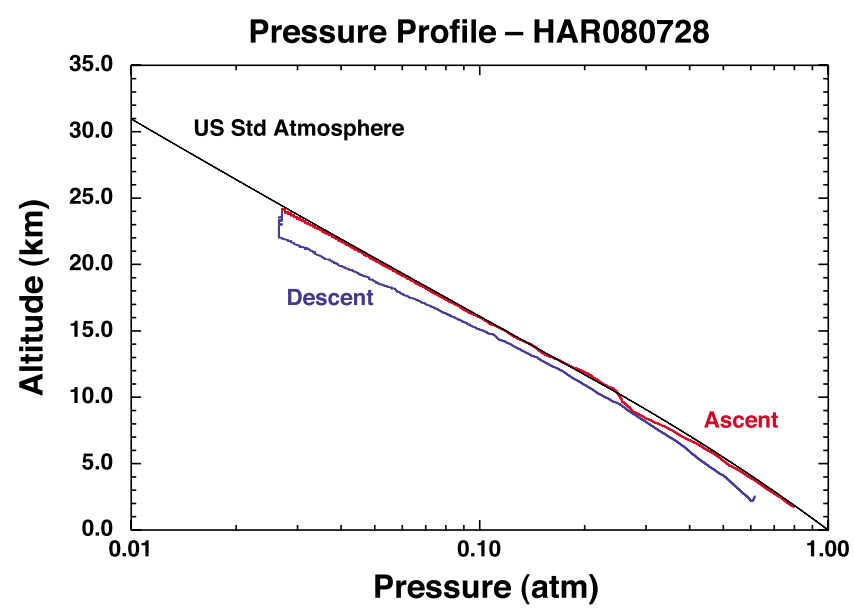

Fig. 9. Pressure versus altitude profiles collected by HARBOR in August 2008, with the overlaid pressure from the U.S. standard atmosphere model (Ref. 5). 


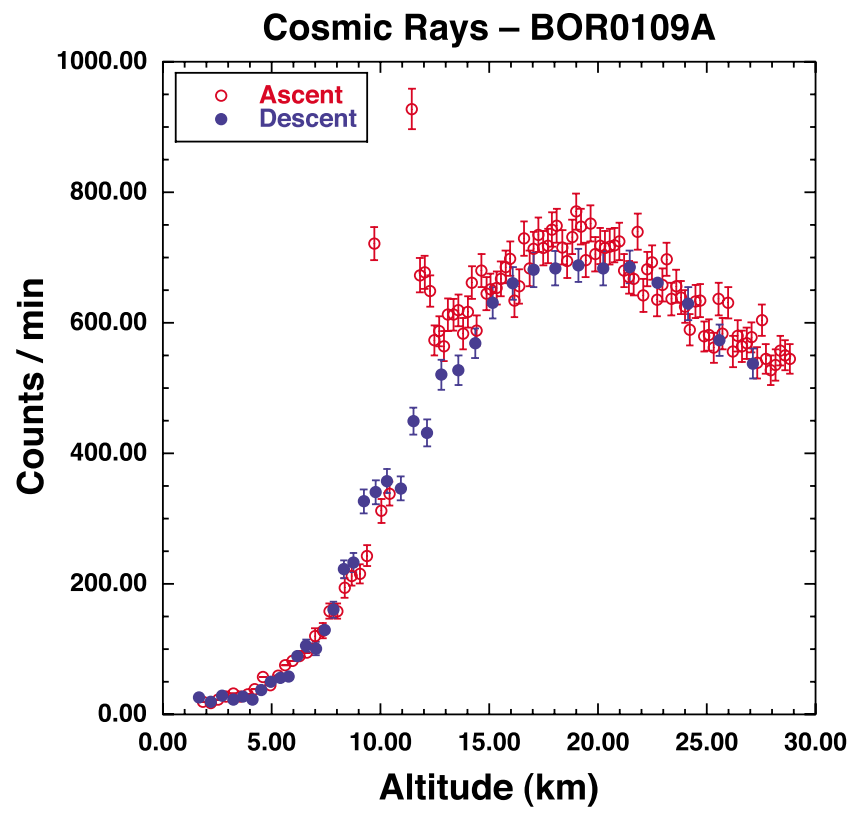

Fig. 10. A cosmic ray data profile collected by BOREALIS in September 2001. The error bars represent $\sqrt{N}$ counting statistics. The turnover at $20 \mathrm{~km}$, the "Pfotzer maximum," is due to the balloon ascending to an altitude above where most secondary cosmic ray showers originate.

$$
P=P_{0} \exp \left[-h / h_{s}\right],
$$

where $P_{0}$ is the surface pressure at zero altitude, and $h_{s}$ is the scale height or e-folding length of the atmosphere. Recorded atmospheric data from a flight can be fit to this model and used to determine the scale height of the atmosphere. For the ascent data shown in Fig. 9, the scale height is found to be $h_{s}=6.58 \mathrm{~km}$.

\section{Cosmic ray profiles}

Between 1911 and 1913, Victor Hess personally flew in balloons to altitudes of $\approx 5 \mathrm{~km}$ to measure the flux of ionizing radiation. He discovered that the flux increased with altitude (at $5 \mathrm{~km}$, the radiation flux was roughly twice its value at sea level), convincing him that the radiation was of cosmic origin. This radiation is now known as cosmic rays and garnered Hess the Nobel Prize in physics in 1936. Off-the-shelf radiation counters allow students to replicate and verify Hess' result and extend the data to much greater altitudes. A typical cosmic ray profile collected by a student flight team with a simple Geiger counter is shown in Fig. 10.

Most cosmic rays detected at the surface of Earth are secondary particles in air showers, created by the collision between a cosmic ray primary and a molecule in Earth's upper atmosphere. Air showers originate at an altitude where the density is high enough that an incoming primary is likely to encounter a target molecule. That altitude is $\approx 20 \mathrm{~km}$, which is seen in the profile of Fig. 10. The increase in particle count is the effect of the many secondary particles in the cosmic ray air showers impinging the detector; the rate drops off after the balloon ascends past most of the secondaries.

\section{Digital imaging}

Photographs are one of the most important returns from any high altitude balloon flight. In addition to being great

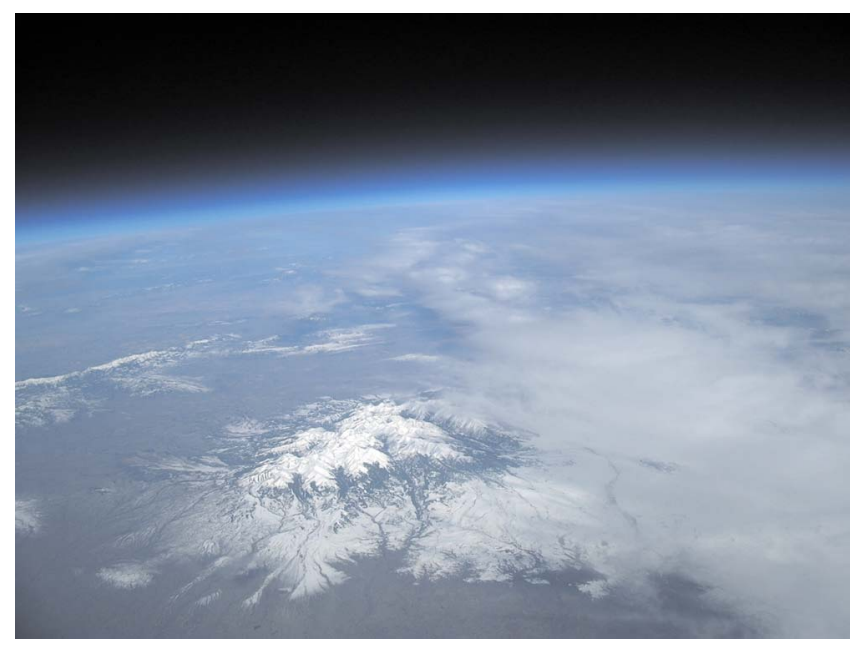

Fig. 11. A typical picture captured in flight by a digital camera system. This view from BOREALIS in April 2005 shows the snow-capped Crazy Mountains of Montana in the foreground, with the curved horizon of Earth and the blackness of space as a backdrop.

vehicles for public relations, photographs make the near space flight experience the most real for students. BOREALIS and HARBOR always fly a digital camera connected to a simple timing circuit that takes approximately 200 to 1000 pictures during the course of a flight (depending on the timing interval between photographs and memory card capacity). A typical image is shown in Fig. 11. The photograph is unretouched and taken with a low-cost compact digital camera. The curvature of Earth is visible, and the sky is black, illustrating that the flight was above most of Earth's atmosphere.

There are two primary analysis activities a student flight team often pursues with a set of high altitude balloon images. The first is geographic identification. With the flight track and good topographic reference maps, this activity can be laboriously done by hand. The time stamp of the photographs can be correlated with the flight data to pinpoint the latitude and longitude where each photograph was taken, and provides a basis for a geo-referencing database that can be incorporated into customized geographic information system software, or geo-referenced software such as Google Earth. Advanced student experiments can include telemetry provided by acceleration or magnetic field sensors; these data will allow image analysts to properly orient the camera references.

A second activity is photogrammetry, the geometrical reconstruction of physical data from the photographs and the properties of the camera. The usual goal of photogrammetry is determining the distance to an object or the altitude of the balloon, or determining the size of an object at a known distance. The geometry of such an analysis is shown in Fig. 12 , where the true object size is $L_{0}$, the image size on the camera's film plane (the CCD in a digital camera) is $L_{i}$, the focal length of the camera is $f$, and the distance to the object of interest is $D$. The analysis of images is based on the geometry of similar triangles, such that the fundamental relation, often referred to as the representative fraction $R_{f}$, is

$$
R_{f}=\frac{f}{D}=\frac{L_{i}}{L_{0}} .
$$

When analyzing digital images, the image size $L_{i}$ is not necessarily known, but it can be expressed in terms of the pixel 


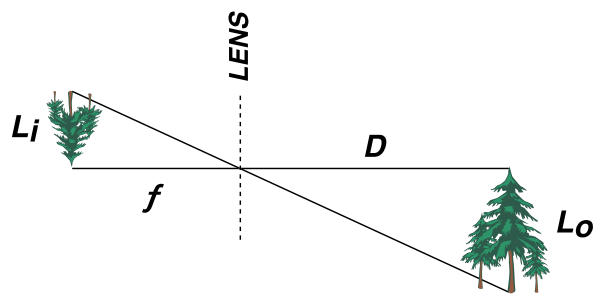

Fig. 12. The basic geometry assumed for photographic analysis, such as the determination of distance to objects and the altitude of the balloon.

size on the CCD, $P_{x}$, in pixels per unit length, and in terms of the size of an object in an image in pixels, $S_{p}$, so that $L_{i}$ $=S_{p} / P_{x}$.

The analysis of images taken at oblique angles can be more complicated depending on the information the flight team desires to extract from the images. More detailed treatments can be found in most texts on remote sensing (for example, Refs. 23 and 24).

\section{E. Other experiments}

The kinds of experiments that can be constructed and flown on a high altitude balloon system are limited only by the constraints of FAA regulations and student ingenuity. The experiments we have described are only a sample of what is possible. Many other experiments could be done, including (but not limited to) atmospheric chemistry, dust and particulate collection (terrestrial and extraterrestrial), ozone monitoring, solar ultraviolet flux versus altitude, variation of $g$ with altitude, upward and/or downward looking video, and infrared digital imaging.

Exploring new scientific experiments also provides new opportunities for students involved in the engineering of flight hardware, particularly in the area of power subsystems, communications and data linking, and microprocessor command and control.

\section{DISCUSSION}

\section{A. The student experience}

In our experience the most important aspect of high altitude balloon programs is they are student driven-the systems are designed, tested, and built by students; the experiments and their objectives are decided by the students; and flight predictions and flight operations are done by the students. Faculty participate, but largely as a source of funds, for mentoring and guidance about the iterative process of designing and flying space hardware, and to enjoy a bit of fun along the way. This student-led approach is distinct from the traditional involvement of undergraduates in research, where the undergraduates act as assistants to a faculty member's research program (Fig. 13).

High altitude ballooning provides hands-on experience building flight systems in an environment that closely mimics the real world. It is a multi-disciplinary endeavor, requiring expertise in engineering, electronics, computer science, and physics. Balloon teams tend to be large, requiring the students to work in groups dedicated to particular subsystems and experiments. Because the experiments and flight systems are often interdependent, the teams must learn how to formulate and connect design reviews and develop rigorous testing procedures for the integrated subsystems. All

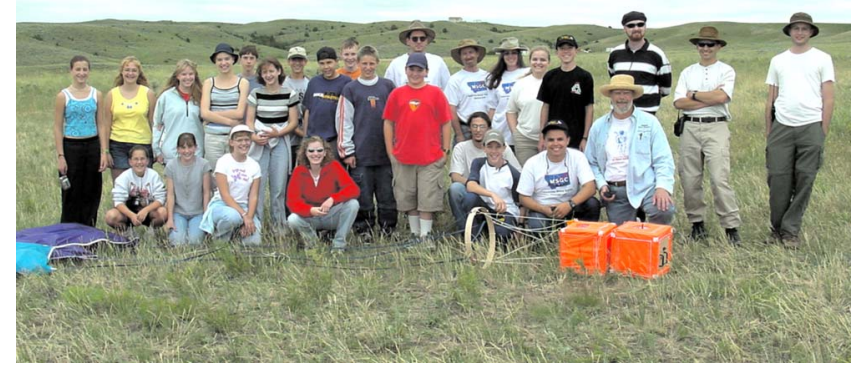

Fig. 13. Recovery team with a BOREALIS parachute-payload stack following a successful flight in June 2001.

these skills and experiences are transferable to careers in aerospace, or entry into higher level education in science and engineering.

\section{B. Before the first flight}

The initiation of a high altitude balloon program is a cause for excitement and will energize a student flight team to action. The development phase in the months before the first flight will often resemble barely contained chaos, but like the flights themselves, provides opportunities for the student flight team to build their skills. One of the most important habits to develop and enforce is the production of documentation-record circuit designs, vendors that provide components and instruments, and the data from test runs that are used to improve system design.

Equally important to keep in mind is that things will go wrong. Flight systems are complicated, and odds are against everything working smoothly and flawlessly. Periodic problems and setbacks are also part of the flight experience, and an important part of the students' education in space hardware development. The students should do their best to minimize problems and mitigate risk during the design phase, but when things go wrong they will have to learn to develop trouble-shooting procedures and make decisions about how to best overcome unexpected issues that arise.

Testing is an important part of maintaining student momentum and excitement during the long run up to the first flight. Ground testing communications hardware and experiments by suspending the stack off buildings, conducting test fills of light-weight $(\approx 1000 \mathrm{~g})$ balloons, and performing drop tests of the parachute system are suitable test activities that will serve to keep your team up and running. Although the cost of a balloon flight is minuscule compared to a rocket launch, it is still worthwhile to do every possible test on the ground before committing hardware to flight-for example, exposing experiments to extreme cold can be as simple as a styrofoam cooler filled with dry ice.

\section{Getting started}

Many balloon programs like HARBOR and BOREALIS exist in the United States and are open to spectators. Visiting and observing flight operations with an experienced team would be highly instructive. Many of these programs have procedures for guest experiments to be flown on their regular flights. Starting in 2002, the Montana and Colorado Space Grant Consortia have held a series of five summer workshops ${ }^{25}$ designed to provide faculty with the knowledge 
and hands-on experience so that they can start a new ballooning program on their campus. A recent survey by NASA's education office reveals that approximately 80 new university-based ballooning programs have been started since 2002 as a result of these workshops.

Funding is often the largest obstacle to beginning a high altitude balloon program. The startup costs are not exorbitant, ranging between $\$ 2000$ and $\$ 3000$, which can often be funded through internal sources. Once the primary balloon hardware has been built, the cost per flight is reduced to the cost of expendables (a new balloon and new helium), roughly \$300-\$400 per flight.

\section{ACKNOWLEDGMENTS}

The authors wish to thank the Montana Space Grant Consortium and the Rocky Mountain Space Grant Consortium for support of these programs, as well as the many students who have worked on the BOREALIS and HARBOR flight programs over the years. The HARBOR program acknowledges the support of the Hemingway Foundation and the Clark Foundation.

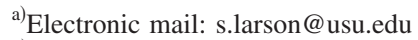

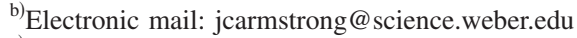

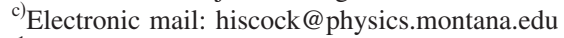

${ }^{1}$ National Research Council, "Issues affecting the future of the U.S. space science and engineering workforce: Interim report" (National Academies, Washington, DC, 2006).

${ }^{2}$ National Research Council, "Building a better NASA workforce: Meeting the workforce needs for the national vision for space exploration" (National Academies, Washington, DC, 2007).

${ }^{3}$ Rising Above the Gathering Storm: Energizing and Employing America for a Brighter Economic Future (National Academies, Washington, DC, 2007).

${ }^{4}$ F. James Rutherford and Andrew Ahlgren, Science for All Americans: Education for a Changing Future (Oxford U. P., New York, 1991).

5 "U.S. standard atmosphere," U.S. Government Printing Office, Washington, DC, 1976), 〈ntrs.nasa.gov/〉.

6 "Federal aviation regulations," U.S. Government Federal Aviation Administration, 〈ecfr.gpoaccess.gov/〉.
}

${ }^{7}$ It is prudent to communicate with the FAA and Air Traffic Control before and during any balloon flight, even if one's payload is technically exempt. Filing a Notice To Airmen a day before flight allows pilots to become aware of the impending flight. One should always be aware of one overarching FAA regulation which applies to all balloon flights: FAR 101.7(a) "No person may operate any moored balloon, kite, unmanned rocket, or unmanned free balloon in a manner that creates a hazard to other persons, or their property." We have always found the FAA to be friendly and helpful. Appropriate, frequent, communication is the key to success.

${ }^{8}$ Hydrogen is a cheaper alternative to helium, but its danger far outweighs the the slight gain in lift and lessening of cost.

${ }^{9}$ L. Paul Verhage, "Near space,"〈www.parallax.com/ $\rangle$.

${ }^{10}$ Edge of Space Sciences Handbook (Edge of Space Sciences, Littleton, CO, 1993), 〈www.eoss.org/handbook/handbook.htm〉.

${ }^{11}$ BOREALIS Handbook, 〈spacegrant.montana.edu/borealis/〉.

${ }^{12}$ Global Forecast System, Air Resource Laboratory, National Oceanic and Atmospheric Administration, 〈www.arl.noaa.gov/ready/〉.

${ }^{13}$ Rock von Glahn, Balloon tracksoftware, 〈www.eoss.org/wbaltrak/〉.

${ }^{14}$ Near space flight track prediction utility, 〈nearspaceventures.com/ w3Baltrak/readyget.pl $>$.

${ }^{15}$ Medgar Evers College high altitude balloon near space education and research explorations, 〈nrts.mec.cuny.edu/mecsat/〉.

${ }^{16}$ M. P. Silverman, "Flying high, thinking low? What every aeronaut needs to know," Phys. Teach. 36, 288-293 (1998).

${ }^{17}$ V. E. Lally, "Balloons for science," Phys. Teach. 20, 438-442 (1982).

${ }^{18}$ R. A. Bachman, "Idealized dynamics of balloon flight," Am. J. Phys. 52, 309-312 (1984).

${ }^{19}$ P. B. MacCready, "Comparison of some balloon techniques," J. Appl. Meteorol. 4, 504-508 (1965).

${ }^{20}$ Balloon Techniques, Part II, Chap. 10 of "WMO Guide to Meteorlogical Instruments and Methods of Observation," WMO-No. 8, World Meteorlogical Organization (2006), 7th ed., 〈www.wmo.int/>.

${ }^{21}$ D. R. Merritt and F. Weinhaus, "The pressure curve for a rubber balloon," Am. J. Phys. 46, 976-977 (1978).

${ }^{22}$ W. H. Press, S. A. Teukolsky, W. T. Vetterling, and B. P. Flannery, Numerical Recipies in Fortran: The Art of Scientific Computing (Cambridge U. P., Cambridge, UK, 1992), 2nd ed., Chap. 3.

${ }^{23}$ J. B. Campbell, Introduction to Remote Sensing, 4th ed. (Guilford, New York, 2007), Chap. 5.

${ }^{24}$ J. R. Jensen, Remote Sensing of the Environment: An Earth Resource Perspective, 2nd ed. (Pearson Prentice Hall, Upper Saddle River, NJ, 2007), Chaps. 3-6.

25 "Starting student space hardware programs," 〈spacegrant.colorado. edu/studentsat/>.

\begin{abstract}
ALL BACK ISSUES NOW AVAILABLE ONLINE
The contents of the American Journal of Physics are available online. AJP subscribers can search and view full text of AJP issues from the first issue published in 1933 to the present. Browsing abstracts and tables of contents of online issues and the searching of titles, abstracts, etc. is unrestricted. For access to the online version of AJP, please visit http://aapt.org/ajp.

Institutional and library ("nonmember") subscribers have access via IP addresses to the full text of articles that are online; to activate access, these subscribers should contact AIP, Circulation \& Fulfillment Division, 800-344-6902; outside North America 516-576-2270 or subs@aip.org.

Individual ("member") subscribers to the print version who wish (for an additional fee) to add access to the online version should contact AAPT or go to the AAPT website: https://www.aapt.org/Membership/secure/agreement.cfm.
\end{abstract}

